Dénoncer les conventionnels pendant la Terreur et la Réaction thermidorienne : des logiques et pratiques entre local et national

Denunciations against the Conventionnels during the Terror and Thermidorian Reaction: rationale and practices between the local and the national

Jean-Baptiste Legoff

\title{
OpenEdition
}

Journals

\section{Édition électronique}

URL : https://journals.openedition.org/ahrf/12779

DOI : 10.4000/ahrf.12779

ISSN : 1952-403X

\section{Éditeur :}

Armand Colin, Société des études robespierristes

\section{Édition imprimée}

Date de publication : 1 juin 2013

Pagination : 81-104

ISBN : 978-2-9083-2789-2

ISSN : 0003-4436

\section{Référence électronique}

Jean-Baptiste Legoff, « Dénoncer les conventionnels pendant la Terreur et la Réaction

thermidorienne : des logiques et pratiques entre local et national », Annales historiques de la Révolution française [En ligne], 372 | avril-juin 2013, mis en ligne le 01 juin 2016, consulté le 01 juillet 2021. URL: http://journals.openedition.org/ahrf/12779; DOI : https://doi.org/10.4000/ahrf.12779 


\section{DÉNONCER LES CONVENTIONNELS \\ PENDANT LA TERREUR ET LA \\ RÉACTION THERMIDORIENNE : \\ DES LOGIQUES ET PRATIQUES ENTRE \\ LOCAL ET NATIONAL}

Jean-Baptiste LEGOFF

Pour beaucoup, Révolution et dénonciation sont intimement liées. La dénonciation, une pratique pourtant fort ancienne, a connu il est vrai un certain renouveau grâce à l'idéologie de la transparence, notamment durant la Terreur et la Réaction thermidorienne. Les représentants du peuple n'ont pas été épargnés par ce phénomène puisque plus de cinq cents lettres ont été écrites pour dénoncer des conventionnels. Ces lettres, dont la plupart ont été envoyées pendant la Réaction thermidorienne, montrent la façon dont les Français perçoivent les événements nationaux à l'aune de leurs expériences sur le terrain. La dénonciation en effet s'inscrit dans cette double appartenance, locale et nationale, et montre la façon dont on a essayé de " sortir de la Terreur » dans certains départements.

Mots-clés : Révolution, dénonciation, délation, Convention nationale, Terreur, Réaction thermidorienne, Représentants du peuple, épuration.

Pierre Larousse, dans son Grand dictionnaire universel, écrivait en 1870, à propos de l'usage de la dénonciation pendant la période révolutionnaire :

« Ainsi qu'on l'avait vu à Rome sous les premiers empereurs, et dans tous les pays aux époques de troubles civils, la délation contribua puissamment à perpétuer les haines intestines. [...] Après le 9 thermidor et sous le Directoire, la représentation nationale fut maintes fois assaillie par des 
récriminations de ce genre, et bien que l'opinion publique se prononçât énergiquement contre la délation, les hommes d'État et les législateurs ne se sentaient pas assez forts pour se passer de ses services ».

À l'instar d'autres périodes troublées, la Révolution aurait donc été un moment propice à la dénonciation. Il est vrai que la pratique de la dénonciation a connu, durant ces années, une certaine vigueur dont témoignent les archives du comité de Législation, des nombreux comités de surveillance et autres institutions révolutionnaires ainsi que l'abondante presse qui fleurit alors.

Les révolutionnaires, dont la plupart ont été formés aux humanités, connaissent tous les sycophantes athéniens ${ }^{1}$ et les delatores de l'Empire romain $^{2}$; ils ont donc en mémoire le souvenir de ces citoyens qui avaient fait de la dénonciation un métier au service de causes souvent peu nobles... La dénonciation fait également partie de la pratique judiciaire de l'Ancien Régime, elle est une composante de son système pénal. À certaines périodes de la monarchie, la dénonciation a même été encouragée par le pouvoir royal $^{3}$, comme elle le sera, dans une certaine mesure, durant la Révolution.

À la veille de la Révolution, la dénonciation est donc une pratique connue mais également décriée, dépréciée.

Les révolutionnaires, pour mettre un terme à ce qu'il convient alors d'appeler l' «obscurantisme » qui a marqué l'Ancien Régime, mettent en avant la transparence, s'inspirant des idées des Lumières ${ }^{4}$. Ainsi, les débats des assemblées sont publics car le peuple peut et doit exercer une surveillance active sur ses représentants. La dénonciation trouve tout naturellement sa place dans l'idéologie révolutionnaire et a ses apologistes comme Mirabeau ${ }^{5}$. Agier, dans le rapport qu'il présente à la commune de Paris le 30 novembre 1789 au nom du Comité des recherches de la

(1) Voir Carine Katarini DogAnIS, Aux origines de la corruption : démocratie et délation en Grèce ancienne, Paris, PUF, 2007.

(2) Voir Yann RIVIÈRE, Les délateurs sous l'Empire romain, Rome, École française de Rome, 2002.

(3) Ainsi, l'édit de François $\mathrm{I}^{\mathrm{er}}$ du 29 janvier 1534 promet une récompense à ceux qui dénonceraient les luthériens ou les personnes qui les aideraient ou les cacheraient.

(4) Certains philosophes, dont Condorcet, pensent en effet que la dénonciation peut être salutaire pour la conduite des affaires publiques en révélant des dysfonctionnements. Voir Charles Walton, Policing Public Opinion in the French Revolution. The culture of Calumny and the Problem of Free Speech, Oxford, Oxford University Press, 2009.

(5) Le $1^{\text {er }}$ décembre 1789, lors de la discussion sur l'organisation des municipalités et la possibilité pour les citoyens actifs de dénoncer les élus municipaux, il déclare : « tout citoyen a droit de dénoncer un crime public. Voilà le principe que toutes les puissances de la terre n'anéantiraient pas. Nous ne pouvons empêcher d'exercer ce droit, je dirai même ce devoir ». AP, t. X, p. 345. 
ville, après avoir déploré le manque de zèle des citoyens de Paris dans la dénonciation des crimes de lèse-nation et le manque d'informateurs, s'écrie :

« Autrefois on abhorrait le personnage de délateur et l'on avait raison, car à quoi aboutissaient les délations ? A faire connaître des actions très innocentes, souvent même vertueuses, et à livrer le prétendu coupable, ou au pouvoir arbitraire, ou à une justice presque aussi redoutable aux gens de bien [...] Aujourd'hui, tout est changé. Ce ne sont plus des actes de vertu ou des démarches indifférentes qu'il s'agit de dénoncer, mais des complots funestes à la Patrie. Le silence, en matière de délation, est une vertu sous le despotisme ; c'est un crime, oui c'en est un, sous l'empire de la liberté » ${ }^{6}$.

La dénonciation, du moment qu'elle est civique ${ }^{7}$, est donc valorisée, et la délation rejetée, mais dénoncer un «complot» en pleine Terreur n'a pas la même signification que durant la Réaction thermidorienne. La dénonciation est donc, pendant la Révolution et plus particulièrement au moment où siège la Convention, pleinement liée aux événements politiques. Comme le rappelle le discours d'Agier, la vision de la dénonciation est malléable car elle dépend des rapports de force politiques : tel dénonciateur « vertueux » peut être vu comme un «vil délateur » quelques mois plus tard.

L'étude que nous avons menée porte sur un peu plus de cinq cents lettres qui ont été envoyées à la Convention ${ }^{8}$. Ces nombreuses dénonciations ${ }^{9} s^{\prime}$ en prennent à ceux qui, de près ou de loin, ont administré la France : soit en étant membre des comités de gouvernement, soit à cause des missions qu'ils ont effectuées, soit encore à cause de leur influence dans leur département d'élection. Tous les représentants ayant siégé à la Convention n'ont pas été dénoncés et tous ne l'ont pas été de la même manière. Ces dénonciations concernent « seulement » cent quatre-vingt dixhuit conventionnels, en premier lieu les anciens représentants en mission et plus particulièrement les montagnards.

(6) Dans Sigismond LaCROIX, Actes de la commune de Paris pendant la Révolution publiés et annotés, [1ere série] t. III, Paris, Cerf, Noblet et Quantin, 1895, p. 77-78. Selon lui, la différence entre dénonciation et délation ne tient pas tant à la motivation du dénonciateur qu'à la nature du régime politique dans lequel est fait la dénonciation.

(7) C'est-à-dire lorsqu'elle est guidée non par un intérêt privé mais par l'intérêt général.

(8) Jean-Baptiste LEGOFF, Représentants face au peuple. Les dénonciations à l'encontre des conventionnels pendant la Terreur et la Réaction thermidorienne, thèse pour le diplôme d'archivistepaléographe soutenue en 2007, dact.

(9) Elles sont essentiellement conservées dans le fonds du comité de Législation qui se trouve aux Archives nationales sous les cotes D III 343-359. 
Durant ces trois années, marquées par l'expérience de la Terreur puis son démantèlement, la vie politique française a été agitée. La guerre civile, avec tout son cortège de proscriptions et de vengeances, est une réalité dans de nombreux départements français. Ailleurs, les luttes de faction sont plus ou moins vives et sont parfois animées par des représentants du peuple en mission porteurs des ordres de la Convention mais devant aussi prendre en compte les données locales et s'insérer dans des réseaux préexistants. Leur action, de ce fait, a été vivement critiquée, c'est pour cela qu'ils sont les principales cibles des dénonciateurs, avant comme après Thermidor.

Longtemps, la Terreur n'a été vue que comme une période où se serait, uniformément, déchaînée une violence d'État contre les ennemis, réels ou supposés, de la République mais, plus récemment, de nombreux historiens ont mis en valeur la diversité des pratiques terroristes sur le territoire français ${ }^{10}$. En allant plus loin, on pourrait aussi dire qu'il n'y a pas une mais des réactions à l'époque thermidorienne. Nous essaierons donc de voir comment les dénonciations, qui donnent à voir cette Terreur sous un autre angle, s'inscrivent dans le paysage politique national et local et comment elles témoignent de cette difficile « sortie de la Terreur».

\section{Les moments de la dénonciation : le poids des événements nationaux}

Toutes les lettres qui ont été recueillies par les différents comités de la Convention ne sont pas datées (environ $15 \%$ du corpus ne comporte aucune indication) mais la présence de cachets de la poste sur un certain nombre de celles-ci permet de situer ces écrits dans le temps.

\section{Dénoncer pendant la Terreur.}

(10) Voir notamment Michel BIARD (dir.), Les politiques de la Terreur, Rennes, Presses universitaires de Rennes, 2008. Ce colloque a fait le point sur les travaux menés sur cette période depuis le bicentenaire de la Révolution française. La Terreur n'est pas envisagée dans cette étude comme un monolithe mais est abordée sous différents angles : violences, politique, économie, culture.. 


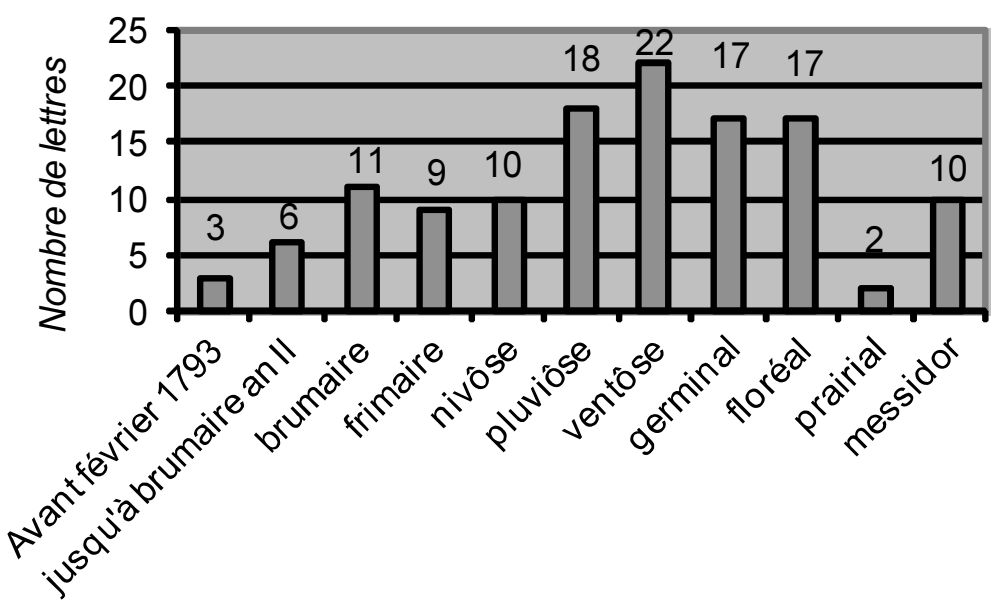

Figure 1. Le graphique ci-dessus présente la répartition des dénonciations entre septembre 1792 et le 9 Thermidor, soit environ un quart du corpus étudié.

Les dénonciations sont rares avant février 1793, c'est-à-dire avant le premier envoi massif de représentants en mars 1793. Avant cette date, seuls quatre-vingt six conventionnels ont été envoyés hors de Paris, le plus souvent pour des missions à caractère militaire ; les représentants sont donc peu en contact avec la population et moins enclins à être la cible de dénonciateurs. On s'attaque alors à des personnages dont l'élection semble entachée d'irrégularités.

En réalité, la vague de dénonciations ne débute véritablement qu'en brumaire an II. Seules six dénonciations sont en effet envoyées à la Convention entre février et le début de l'an II. Les mois de mars et d'avril sont le moment où la Convention crée véritablement la fonction de représentant du peuple en mission : les envois se font plus massifs et les contours de leurs pouvoirs se dessinent. L'été 1793 est également le moment de tous les dangers, tant intérieurs qu'extérieurs, et les représentants du peuple en mission jouent un grand rôle dans le rétablissement de la situation sur de nombreux fronts. Une fois la fièvre retombée, les langues commencent à se délier alors même que le nombre de commissaires dans les départements ou aux armées a atteint son maximum ${ }^{11}$.

De plus, en cet automne 1793 l'heure est aux règlements de comptes tant dans les régions fédéralistes que dans les zones d'insurrection royaliste. C'est ce qui explique la véritable envolée du nombre de lettres qui

(11) Sur les représentants en mission, voir Michel BIARD, Missionnaires de la République, les représentants du peuple en mission, 1793-1795, Paris, CTHS, 2002. 
s'attaquent aussi bien aux prétendus fédéralistes ou aristocrates qu'à ceux qu'on nomme déjà les indulgents.

Le pic de la pratique dénonciatrice pré-thermidorienne se situe entre les mois de pluviôse et de floréal, c'est-à-dire au moment où la lutte des factions bat son plein à Paris. On fustige alors des modérés - des indulgents - mais aussi des ultra-révolutionnaires. Dans l'Ain par exemple, Gouly est critiqué parce qu'il a fait mettre en liberté des suspects alors que son successeur, Albitte, subit les foudres de ceux qui acceptent mal la mise en place de certaines mesures terroristes et la déchristianisation orchestrée par le représentant du peuple ${ }^{12}$.

On assiste à un certain refroidissement de l'ardeur dénonciatrice en prairial. Il est vrai que le Comité de salut public a rappelé à Paris de nombreux représentants du peuple en mission, notamment les plus en vue (Carrier, Fréron, Tallien, Fouché, Albitte, Javogues...). Peut-être aussi peut-on y voir une certaine prudence de la part des Français car dénoncer quelqu'un après les épurations du printemps et les lois de prairial n'a pas la même signification, ni les mêmes implications, que dénoncer quelqu'un au début de 1793.

Ce que les historiens ont nommé la Grande Terreur coïncide donc avec un quasi-arrêt des dénonciations bien qu'un certain nombre de lettres soient envoyées en messidor, soit pour dénoncer un « proconsul » encore en place (en l'occurrence Le Bon principalement ${ }^{13}$ ), soit pour dénoncer des faits plus anciens (Dubois-Crancé et Gauthier sont critiqués pour leur rôle équivoque lors du siège de Lyon).

\section{La « Révolution du 9 Thermidor » et la libération de la parole dénonciatrice.}

Thermidor constitue véritablement une fracture dans la pratique dénonciatrice, car la plupart des lettres $(75 \%)$ ont été envoyées à la suite de la mise à mort de Robespierre et de ses supposés « complices ».

(12) AN D III 343-344, dossiers « Albitte, Amar, Javogues, Méaulle » et « Albitte et autres »; AN D III 349-350, dossier « Gouly ».

(13) Le Bon, le plus dénoncé des conventionnels, est un cas un peu atypique puisqu'il cumule le fait d'avoir accompli une longue mission (durant l'année où il siège à la Convention, il est pendant neuf mois en mission), d'avoir été envoyé dans son département d'élection (il est ainsi face à des dénonciateurs qui le connaissent bien) et d'avoir eu pour ennemi un conventionnel, Guffroy, lui aussi élu du Pas-de-Calais et réacteur ardent. Son cas est donc particulier, bien que proche de celui de Javogues et de Le Carpentier (qui font eux aussi partie des plus dénoncés), mais emblématique de la complexité du phénomène dénonciateur qui ne tient pas seulement à l'ampleur des faits dénoncés mais aussi à l' «exposition » politique de celui qu'on accuse. 


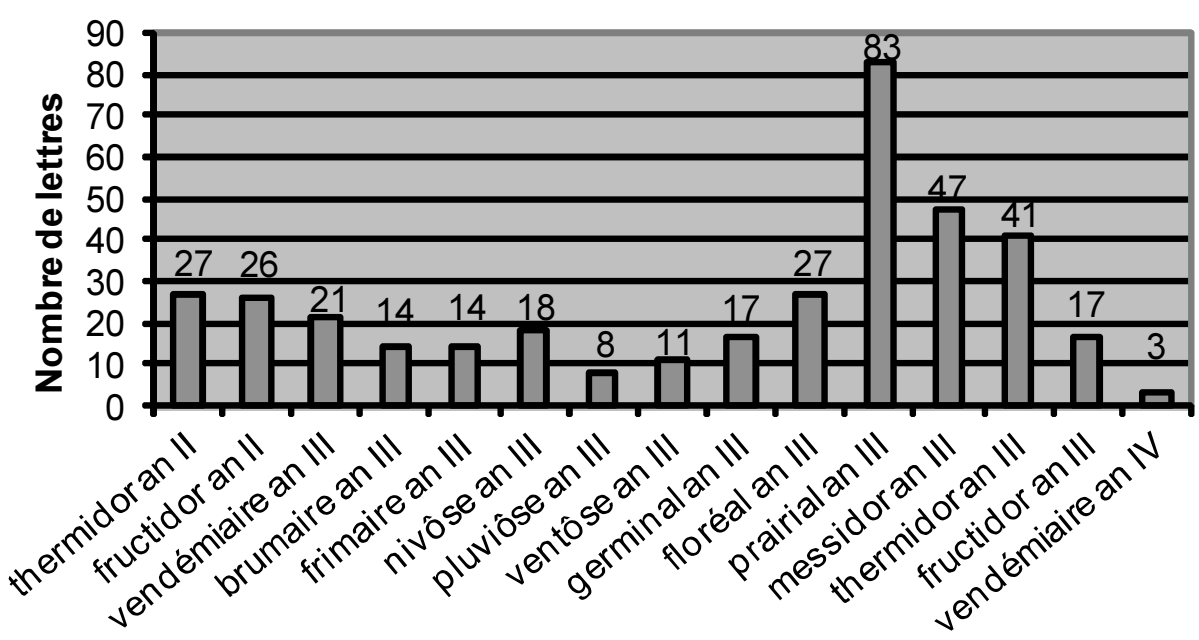

Figure 2. Le graphique ci-dessus représente le nombre de lettres envoyées après Thermidor.

Les derniers mois de l'été correspondent à une véritable libération de la parole. Les dénonciateurs semblent s'être pleinement appropriés les paroles que Réal prononce à la tribune des Jacobins à sa sortie de prison :

«Pour bien détester le régime qui vient de finir, je crois qu'il est nécessaire d'en faire voir ses dégoûtants effets. C'est dans la peinture de maux que l'on faisait souffrir dans les prisons que l'indignation doit trouver son aliment. [...] Je ne crois pas, comme on l'a dit dans certains rapports, que la Révolution soit une vierge dont on ne doit pas lever le voile $»^{14}$.

Cet appel à la révélation, à la dénonciation, semble avoir été bien compris dans les départements et on dénonce à qui mieux mieux la «queue de Robespierre $»^{15}$, ses « continuateurs $» .$. Ainsi, dans une dénonciation contre Le Bon, le conseil général de la commune de Cambrai écrit : « Nous serions indignes d'êtres libres, nous deviendrions criminels si nous taisions des faits qui ont excité nos inquiétudes ; le salut de la patrie nous fait un devoir suprême de les faire connaître $»^{16}$.

(14) Cité par Bronislaw BACZKO, Comment sortir de la Terreur? Thermidor et la Révolution, Paris, NRF essais, Gallimard, 1989, p. 192-193.

(15) Voir Michel BIARD « Après la tête, la queue ! La rhétorique antijacobine en fructidor an II - vendémiaire an III », dans Le Tournant de l'an III, réaction et terreur blanche dans la France révolutionnaire, CTHS, 1997, p. 201-214

(16) $\mathrm{AN} \mathrm{F}^{7} 4772$, liasse B, lettre du 17 thermidor an II (4 août 1794). 
La chute des triumvirs n'est donc pas seulement perçue comme la mise à l'écart brutale d'une nouvelle faction, une sorte de coup d'État, mais bien comme un changement politique majeur. En effet, les précédentes luttes de faction n'avaient pas autant mobilisé les plumes. Certains dénonciateurs n'hésitent pas à inviter la Convention à aller plus loin dans l'épuration car les complices de ceux qui ont été exécutés le 10 thermidor sont, selon eux, encore actifs. Dans une lettre du 15 thermidor (2 août), un anonyme félicite la Convention pour son œuvre tout en lui suggérant d'aller plus loin dans la punition des complices de Robespierre :

«Citoïens sauveurs de la patrie, vrais restaurateurs de la liberté. Les victorieuses batailles de Gemmappe et Fleurus n'ont jamais excité une allégresse aussi universelle que celle que vous venez de remporter sur le scélérat et tirannicide Robespierre. Vous avez abattu la tête altière de l'arbre mais ses rameaux s'étendent sur tous les départements. Veillés citoïens, et vous trancherez toutes ses ramifications $»^{17}$.

Beaucoup d'anciens représentants en mission terroristes font les frais de cette flambée dénonciatrice : Le Carpentier d'abord (il est encore en mission dans la Manche), Rovère, Borie (le « Robespierre du midi » selon ses détracteurs), Le Bon, Maignet, Laignelot, Bo... Un certain nombre de ceux qui sont alors dénoncés n'avaient pas été inquiétés avant le 9 Thermidor, ce qui montre que la chute de Robespierre est bien perçue comme un renouvellement, une « réaction » (au sens premier du terme). Il s'agit alors pour les dénonciateurs de faire de leur cible des complices de ceux que la Convention a fait périr sur l'échafaud.

\section{De brumaire à germinal : la versatilité de la pratique dénonciatrice.}

À la fin de l'été, les esprits s'apaisent ${ }^{18}$, comme si l'opinion cherchait à deviner les intentions d'une Convention qui hésite encore à s'en prendre aux terroristes, comme en témoigne notamment la panthéonisation de Marat le 26 fructidor (12 septembre), et qui lance un appel au pardon et à l'oubli le $4^{\mathrm{e}}$ sans-culottide an II (20 septembre).

(17) AN D III 352, dossier « Le Carpentier », lettre du 15 thermidor an II (2 août 1794) envoyée de Coutances (Manche).

(18) Vendémiaire est un moment particulier car sur les vingt-et-une dénonciations envoyées à la Convention, neuf visent Carrier que les réacteurs tentent alors désespérément de faire juger et que la Convention semble prête à sacrifier. De plus, Le Bon, qui a officiellement été mis en cause par la Convention et arrêté le 15 thermidor an II (2 août 1794), est lui aussi dénoncé. Le grand nombre de dénonciations envoyées n'est ainsi dû qu'à la poursuite des dénonciations contre les deux «proconsuls ». 
Brumaire, frimaire et nivôse constituent une période assez « calme » (par rapport aux derniers mois de l'été) : le nombre de dénonciations envoyées à la Convention baisse quelque peu. C'est en effet l'époque où les réacteurs imposent leur politique au sein de la Convention : la société mère des Jacobins est fermée le 22 brumaire an III (12 novembre 1794) et Carrier est envoyé devant le Tribunal révolutionnaire pour son action à Nantes le 3 frimaire ( 23 novembre). Cette période constitue donc un véritable tournant qui marque véritablement l'entrée dans la « Réaction», c'est-à-dire la remise en cause du passé terroriste, au moins dans l'opinion publique. Durant ces deux mois, pendant lesquels l'opinion publique est particulièrement sollicitée par les pamphlets et journaux qui relatent les crimes de Nantes, la plupart des lettres écrites s'attaquent à d'anciens terroristes.

Bronislaw Baczko a souligné, à juste titre, l'importance cruciale du procès de Carrier dans l'évolution politique de la Convention en l'an III :

« D'autres procès suivront, dont celui de Fouquier-Tinville (germinalfloréal an III). Jamais ils n'influeront autant que le firent les procès du comité révolutionnaire de Nantes et de Carrier, le tour que prit la sortie de la Terreur. [...] Ils contribuèrent à créer contre les Jacobins et le personnel terroriste un sentiment d'horreur inexpiable. [...] Les procès légitimaient, en quelque sorte, le droit à la vengeance $»^{19}$.

D'ailleurs, dans les mois qui suivent, l'exemple de Carrier est repris par un grand nombre de dénonciateurs : qui pour comparer la personne dénoncée au député du Cantal, tel autre pour rappeler que l'exécution de Carrier n'a de sens que si ses « complices » ou « imitateurs » sont à leur tour exécutés. La manœuvre de la Convention, ayant consisté, selon les mots de Sergio Luzzatto, à « se hâter d'incriminer Carrier, député-symbole, afin de freiner la dérive contre-terroriste $»^{20}$ se révèle un échec. Ainsi, les citoyens de la commune de Lyon déclarent-ils à la Convention à propos de Collot-d'Herbois : « [il] s'est jugé lui-même en prononçant contre son digne émule, l'exécrable Carrier ! ${ }^{21}$. De même, les citoyens de la commune de Belley (Ain) citent le discours prononcé par Albitte lors du vote sur la

(19) Bronislaw BACZKO, Comment sortir de la Terreur?, op. cit., p. 206.

(20) Sergio LuZZATTO, L'Automne de la Révolution, luttes et culture politique dans la France thermidorienne, traduit de l'italien par Simone Carpentari Messina, Paris, Honoré Champion, 2001, p. 41.

(21) $\mathrm{F}^{7} 4438$, plaquette 7 , pièces 275 et 276 , adresse des citoyens de la commune de Lyon lue à la Convention le 17 ventôse an III (7 mars 1795). 
mise en accusation de Carrier dans lequel le représentant reproche à son collègue du Cantal d'avoir usé de moyens « effrayants » qui n'ont servi à rien. Ayant rappelé ces paroles ils s'adressent à la Convention en lui demandant : "Quel sort mérite celui, qui n'ayant ni guerre civile à terminer, ni rébellion à punir, ni aucun des motifs au moins spécieux sur lesquels Carrier pouvoit fonder une excuse ; s'est cependant permis d'employer les mêmes mesures et de l'égaler en cruautés ? $\gg^{22}$. Alors qu'une partie de la Convention semble espérer que la punition de Carrier va calmer les esprits et mettre un terme à la demande de justice et de vengeance, certains, ils sont nombreux, pensent au contraire que ce procès et cette exécution ne sont qu'un début : d'autres conventionnels doivent subir le même sort.

«L'affaire Carrier », si elle influence l'opinion publique, joue aussi un rôle dans la mise en place d'un cadre légal à la pratique dénonciatrice et plus largement à la mise en accusation d'un représentant du peuple. Depuis la suppression, dès le 15 thermidor (2 août 1794), de la loi qui permettait aux Comités de salut public et de sûreté générale de mettre en arrestation un représentant sans qu'il ait été entendu il existe un vide juridique. Celui-ci va vite se faire pesant du fait du nombre croissant de dénonciations qui parviennent à la Convention et surtout de la mise en jugement des commissaires révolutionnaires de Nantes ${ }^{23}$. Ces derniers ne nient pas les faits qui leur sont reprochés mais prétendent que le seul responsable en est Carrier. Ils demandent à ce qu'il soit lui aussi jugé. La Convention doit agir, d'autant plus que l'opinion publique, au moins à Paris, demande elle aussi la punition de Carrier et des commissaires, dont les crimes sont connus par les journaux qui relatent au jour le jour les récits faits devant le Tribunal. Pourtant, les conventionnels sont divisés quant à la manière de mettre en accusation un représentant du peuple : Dumont voudrait que ce soient les comités qui prennent cette décision alors que Tallien prétend que ce pouvoir ne peut appartenir qu'à l'Assemblée. Merlin (de Douai) dépose un rapport sur cet objet le 2 brumaire ( 23 octobre) mais tous savent que le décret sur les procédures de mise en accusation d'un député sera immédiatement applicable à l'élu du Cantal. C'est au terme de longs débats que la Convention vote, le 8 brumaire (29 octobre), un décret définissant les modalités de mise en accusation d'un député. Il s'agit en fait d'une procédure lente et complexe. Elle concerne dans un premier

(22) AN D III 343, dossier « Albitte et autres », s. d.

(23) Ouvert le 25 vendémiaire an III, ce procès conduit à la mise en accusation de Carrier. Cf. Bronislaw BACZKO, Comment sortir de la Terreur, op. cit., p. 191-254 et Corinne GoMEZ-LE Chevanton, Carrier et la Révolution en 30 questions, La Crèche, Geste éditions, 2004, p. 42-51. 
temps les Comités de salut public, sûreté générale et législation réunis qui examinent les dénonciations envoyées à la Convention. S'il y a lieu, ils soumettent le cas à la Convention qui nomme, par tirage au sort, une commission de 21 membres chargée d'enquêter sur les faits dénoncés. Si la commission conclut à la mise en accusation, celle-ci est mise aux voix par appel nominal. Le même jour, une commission de 21 membres est instituée pour statuer sur le cas de Carrier, elle rend ses conclusions le 21 brumaire (11 novembre).

Durant cet automne agité, les Crétois, ceux qui sont restés fidèles aux idéaux de l'an II à l'Assemblée, sont marginalisés. Beaucoup d'anciens terroristes prennent en effet le parti de la «Réaction » à la suite de Tallien, Fréron ou encore Dumont. Pourtant, si les anciens terroristes sont la cible privilégiée des dénonciateurs au début de l'an III, les choses changent quelque peu en nivôse puisque sur les dix-huit lettres qui parviennent à la Convention, neuf s'en prennent à des « réacteurs », accusés de profiter du 9 Thermidor (car personne ne remet jamais en cause la justesse de l'exécution des triumvirs) pour asseoir leur pouvoir personnel ou même pour faire triompher le royalisme. Charles Delacroix est ainsi taxé de royalisme par un citoyen détenu dans les Ardennes ${ }^{24}$ tandis que le citoyen Vassis, qui se présente comme un sans-culotte persécuté par les fédéralistes, s'en prend à Legendre et tous les « réacteurs ». Il voit dans ce nouveau «parti » un danger pour la Révolution : « Je parle à la faction naissante, je vais dépeindre leurs désirs et l'ambition qui les gouvernent ; perres [sic] du vrai peuple méfiés-vous [sic] » et il ajoute : «Combien avez-vous vendu les droits de l'homme? A quel prix livrerés-vous [sic] la Constitution républicaine tas de fripons, voyous $\gg^{25}$. La politique de la majorité de la Convention ne fait donc pas l'unanimité même si le rejet de la Terreur domine largement dans les lettres. Le conformisme politique de façade (nul ne se risque en effet à faire l'éloge de Robespierre) n'empêche donc pas les dénonciateurs de critiquer certains des aspects de la politique de la Convention.

L'unanimité initiale se fissure, ce qui se voit aussi dans les adresses qui sont alors envoyées à la Convention et qui se plaignent du modérantisme qui refait surface (la première est envoyée par la société populaire de Dijon

(24) AN D III 346, dossier « Delacroix », lettre du citoyen Mogue, Sedan (Ardennes), 14 nivôse an III (3 janvier 1795).

(25) AN D III 352, dossier « Legendre, Lacroix et Louchet », Chagny (?), 15 nivôse an III (4 janvier 1795). 
dès le 7 fructidor). Désormais, deux camps aux contours flous ${ }^{26}$ se dessinent : ceux qui veulent aller plus loin dans la Réaction et ceux qui restent attachés à certains des principes de l'an $\mathrm{II}^{27}$.

Car les dénonciateurs restent très attentifs à ce qui se passe à Paris et dans l'Assemblée. Chaque événement est connu, commenté et analysé par ceux-ci. Ainsi, alors que le 15 frimaire an III (5 décembre 1794), la Convention charge ses comités d'enquêter sur les anciens membres des comités de l'an $\mathrm{II}^{28}$ et qu'une commission de 21 membres est nommée le 21 nivôse (10 janvier), nombreux sont les dénonciateurs qui s'en prennent à ceux qu'on appelle les « Quatre». On ne saurait y voir l'effet d'un hasard ; beaucoup semblent avoir attendu ce signal politique pour agir.

Pourtant, le nombre de dénonciations stagne durant les mois de pluviôse et ventôse. Ce reflux modéré de la verve dénonciatrice trouve sans doute son explication dans les tergiversations d'une Assemblée qui disculpe Maignet et fait traîner les travaux de la commission de 21 membres, laissant ainsi penser que l'heure n'est plus aux règlements de comptes. De plus, en cette fin d'hiver, une grave disette touche la France. L'urgence n'est donc plus d'épurer mais de gérer les subsistances. Les seules lettres qui parviennent alors à la Convention s'en prennent aux anciens membres des comités de l'an II ainsi qu'à des terroristes, comme si leurs auteurs voulaient signifier à la Convention leur détermination à poursuivre l'épuration. Certains d'ailleurs n'hésitent pas à critiquer cette lenteur que met l'Assemblée à poursuivre les anciens terroristes. Le citoyen Messigeon qui s'attaque à Billaud-Varenne, Collot-d'Herbois, Barère, Vadier et Duhem écrit ainsi en pluviôse : «Quel reproche la Convention n'a-t-elle pas déjà à ce [sic] faire d'avoir laissé tous ces crimes impunis puisque ces coquins en voyant que l'on les laisse impuni lève [sic] la tête partout $»^{29}$. Selon lui donc, et ce n'est pas le seul à professer une telle maxime, seule la punition des coupables permettra de pacifier la France, c'est-à-dire de finir la Révolution.

(26) Ainsi, certains de ceux qui ont contribué à la chute de Robespierre comme Fouché feront les frais de la Réaction (il est arrêté à l'été 1795) alors que certains des « réacteurs » de la première heure, tel Babeuf, prendront leur distance vis-à-vis de la Réaction.

(27) Selon Françoise Brunel, les derniers Montagnards ne se rendent compte du tour que prend la réaction qu'à l'hiver de l'an III, eux qui ne voyaient dans le 9 Thermidor qu'un nouveau 31 mai. Françoise BRUNEL, «Les derniers Montagnards et l'unité révolutionnaire », AHRF, n 229, juillet-septembre 1977, p. 392.

(28) À savoir Collot-d'Herbois, Vadier, Barère et Billaud-Varenne.

(29) $\mathrm{AN} \mathrm{F}^{7} 4443$, plaquette 3, pièce 99, Nantes (Loire-Inférieure), s. d. La dénonciation a été reçue à la Convention le 20 pluviôse an III (8 février 1795). 


\section{L'effet catalyseur des journées de germinal et prairial.}

Encore une fois, la vague dénonciatrice va être relancée par les événements nationaux puisque les émeutes des 12 et 13 germinal an III $\left(1^{\text {er }}\right.$ et 2 avril 1795) et, surtout, les troubles de prairial (du $1^{\text {er }}$ au 4$)$, vont susciter un véritable déferlement de dénonciations.

Il faut dire que la Convention donne alors l'exemple : dix-huit députés sont jetés en prison après le 12 germinal et vingt-cinq dans les jours qui suivent les événements de prairial ${ }^{30}$. Durant ces séances, l'Assemblée se mue en une véritable arène. Fréron, habile orateur, s'écrie lors de la mémorable séance du 12 germinal : «ils [les émeutiers et leurs soutiens] ont cru noyer dans le sang la révolution du 9 thermidor ; mais non, elle va recevoir son complément $»^{31}$. En ces heures troubles tout le monde sait que ce « complément » signifie l'arrestation de tous ceux qui ont été, à un degré ou un autre, les « complices de Robespierre », c'est-à-dire les anciens terroristes et les Crétois.

Les mesures répressives ne se limitent pas à l'enceinte de la Convention: partout les terroristes sont désarmés tandis que les émigrés du 31 mai sont invités à revenir en France... la Terreur blanche fait rage dans certains départements. L'heure n'est donc plus à l'oubli, encore moins au pardon, et les passions se déchaînent.

Les émeutes de germinal et de prairial ont eu un effet catalyseur indéniable dans la pratique dénonciatrice. Dans de nombreuses lettres, on retrouve l'idée que les citoyens doivent aider la Convention à s'épurer en lui faisant connaître les «membres gangrenés » qui y siègent encore. À en croire les dénonciateurs, c'est l'amour du bien public qui les guide, et non la vengeance ${ }^{32}$.

Après les arrestations décidées dans la tourmente en germinal, la Convention réfléchit à la façon de poursuivre cette épuration, le code de brumaire n'étant plus jugé satisfaisant du fait de sa lenteur. Elle décide le 21 floréal (10 mai 1795) que les Comités de salut public et de sûreté générale ne seront plus compétents pour examiner la conduite des représentants du peuple et charge le comité de Législation de réfléchir au moyen de faire cet examen $^{33}$. Le 20 floréal an III (9 mai 1795), lors d'une discussion sur la

(30) Voir Françoise BRUNEL, « L'épuration de la Convention nationale en l'an III », dans Le Tournant de l'an III, op.cit., p. 15-26.

(31) Moniteur, t. 24, $\mathrm{n}^{\circ}$ du 15 germinal an III, p. 120.

(32) Le fait que de nombreux dénonciateurs cherchent à se justifier montre assez que la dénonciation, loin d'être un acte banal, est encore entachée d'infamie.

(33) AN D III 343-344, Décret de la Convention. Il s'agit en fait d'une mesure s'inscrivant dans un contexte plus large de réorganisation des comités datant du 15 floréal. Le Comité de salut 
réorganisation du gouvernement révolutionnaire, le représentant Villetard déclare :

«Il faut assurer au peuple la possibilité de dénoncer un de ses représentants. La liberté publique et individuelle réclament cette garantie ; mais elles réclament en même temps et au moins aussi impérieusement la liberté la plus entière d'opinion pour chacun des représentants du peuple $»^{34}$.

Il fait ainsi ressurgir un débat aussi vieux que la Révolution qui a animé nombre de discussions au sein des différentes assemblées à savoir : quelle est la frontière séparant droit à la dénonciation et répression de la calomnie $^{35}$ ? La nécessaire censure du peuple ne saurait être aveugle : beaucoup voudraient que les représentants du peuple bénéficient de garanties car tous savent qu'ils sont susceptibles d'être la cible de dénonciations. Le comité de Législation semble d'ailleurs peu pressé puisqu'il ne présente aucun projet sur les moyens de faire l'examen des députés dénoncés. Tout en favorisant l'envoi de dénonciations, la Convention se montre donc prudente et semble réticente, dans sa majorité, à mettre un œuvre un examen généralisé de la conduite des représentants ${ }^{36}$.

Cette indécision n'est plus d'actualité au lendemain des émeutes de prairial et les dénonciations qui « dormaient » jusque-là dans les archives des comités refont surface. Le discours prononcé le 5 prairial an III (24 mai 1795) par André Dumont, hier terroriste et aujourd'hui ardent réacteur, est symptomatique de ce désir de «purification » et de ce climat de peur, feint ou réel, qui affecte une partie de la population après ces événements ; il s'écrie en effet :

« Puisque le danger n'est pas encore passé, puisqu'il ne faut pas oublier le mot d'un de nos décemvirs : «Le lion dort ; il pourra se réveiller ${ }^{37}$ »,

public retrouvant à cette occasion un grand nombre de pouvoirs, la Convention juge utile de lui retirer l'examen de la conduite des députés car le souvenir de l'an II, lorsque le «Grand comité » pouvait faire arrêter les députés, hante les esprits.

(34) Moniteur, t. 24, n $^{\circ}$ 233, 23 floréal an III, p. 422.

(35) La question de la liberté de la presse, liée à celle de répression de la calomnie, a divisé les députés de l'Assemblée constituante puis de la Législative. Voir Charles WALTON, Policing Public Opinion, op. cit.

(36) Ainsi, la Convention tire au sort, le 17 floréal an III (7 mai), une commission de 21 membres chargée d'enquêter sur le cas de Le Bon, en prison depuis thermidor an II. Elle ne rend ses conclusions que le 22 messidor (10 juillet) et Le Bon est alors traduit devant le tribunal criminel de la Somme qui le condamnera à mort. Il peut être surprenant de voir comment la Convention qui ne s'est pas embarrassée de respecter les formes légales pour faire arrêter certains de ses membres en germinal et prairial tient tant à suivre à la lettre le code de brumaire an III.

(37) Il s'agit d'une citation, approximative, des paroles prononcées par Billaud-Varenne au club des Jacobins le 13 brumaire an III (3 novembre 1794) : « le lion n'est pas mort quand il sommeille, 
prenons toutes les mesures convenables pour prévenir ce funeste réveil, qui peut causer la perte de la liberté. On s'occupe dans les sections de purger Paris des hommes qui ont servi la tyrannie ; occupons-nous de découvrir ceux qui sont encore dans votre sein. (On applaudit). Plus de mesures partielles, plus de clémence pour les assassins de la patrie. [...] Je demande que vous fixiez l'époque où vous entendrez le rapport des comités que vous avez chargés de recueillir toutes les dénonciations faites contre les représentants qui, par des méfaits quelconques, auraient déshonoré leur caractère $»^{38}$.

Cette mesure adoptée par l'Assemblée permet au comité de Législation d'enquêter sur les missionnaires qui ont été envoyés dans les départements ou aux armées durant la Terreur. Désormais les dénonciations serviront aux enquêtes menées par le comité de Législation.

Aux dénonciations parvenues à la Convention depuis des mois, s'ajoutent de nombreux écrits. Cette recrudescence de la pratique dénonciatrice n'est sans doute pas étrangère à la publication de ce décret qui semble inviter les «bons » citoyens à déposer leurs plaintes au Comité. Ainsi, un caporal dénonçant Laignelot et Lequinio écrit que : «par ce décret la Convention semble faire un appel à la conscience des citoyens ${ }^{39}$.

En effet, un grand nombre de dénonciations sont envoyées durant les deux premières décades de prairial puis le rythme s'essouffle quelque peu dans la dernière décade de ce mois avant de chuter lentement à partir de la deuxième décade de messidor. L'arrestation d'un certain nombre des terroristes les plus en vue (Albitte, Le Carpentier, Pinet aîné, Javogues...) explique sans doute cette baisse, toute relative, du nombre de dénonciations. Peut-être aussi que les débuts de la Terreur blanche qui secoue le Midi font revivre un ennemi que les révolutionnaires avaient eu tendance à oublier, le royalisme, ce qui éclipse quelque peu la peur du jacobinisme. Le débarquement de Quiberon, le 9 messidor (27 juin), alarme les thermidoriens qui craignent un retour des émigrés. L'urgence

et à son réveil il extermine ses ennemis » (Alphonse AULARD, La Société des Jacobins. Recueil de documents pour l'histoire du club des Jacobins de Paris, t. VI, Paris, Cerf, Noblet et Quantin, 1897, p. 633). Ces paroles, largement reprises par la presse, valent à son auteur la haine des thermidoriens qui l'ont prise comme prétexte à leur demande de vengeance et d'épuration.

(38) Moniteur, $\mathrm{n}^{\circ}$ 250, 10 prairial an III (29 mai 1795), p. 546.

(39) AN D III 351, dossier « Laignelot et Lequinio »; lettre de Rivière jeune, caporal fourrier, Rochefort (Charente-Inférieure), 15 prairial an III (3 juin 1795). 
est au rassemblement de tous les partisans de la Révolution, fussent-ils jacobins, comme le rappelle Doulcet ${ }^{40}$.

\section{L'été 1795 : les derniers règlements de compte.}

Après la baisse du début de l'été, on observe une certaine reprise de la pratique dénonciatrice à partir de la deuxième décade de thermidor et jusqu'au début de fructidor. Ce sursaut s'explique sans doute par l'attente du dernier rapport que doit faire le Comité de législation ${ }^{41}$. Certains pensent que le vote de la Constitution doit être le moment pour la Convention de s'épurer définitivement, comme l'exprime un cultivateur de la Somme : « la Convention veut que ceux qui discuteront l'acte constitutionnel soient recommandables par leur pureté et leur intégrité, en ce cas il y aura bien peu de ceux de vos collègues qui ont été en mission qui participeront à la formation de cette constitution $»^{42}$.

Durant l'été, si les terroristes sont encore la principale cible (on s'attaque à ceux qui n'ont pas été arrêtés mais aussi à ceux qui sont en prison et dont on attend le jugement), d'autres conventionnels sont fustigés pour leur patriotisme « douteux ». Lanjuinais, Laurence, Saladin, Delamarre, Cadroy... sont accusés de favoriser les royalistes et de faire le jeu de la contre-révolution. L'appel de Doulcet semble donc avoir été entendu par certains.

Une fois la dernière épuration passée et la Constitution votée le 5 fructidor (22 août), le flot se tarit. Les dénonciateurs n'ont en effet plus beaucoup d'espoir de voir leurs plaintes aboutir.

On le voit, les dénonciateurs sont donc attentifs aux événements nationaux ainsi qu'aux décisions de la Convention, ils ne prennent pas la plume à n'importe quel moment et certains épisodes politiques majeurs libèrent la parole à l'instar du 9 Thermidor ou des émeutes populaires

(40) Le 13 messidor il fait un rapport sur le débarquement de Quiberon et s'exclame : « Ce n'est pas au moment où le terrorisme est comprimé et où vos comités de gouvernement, pénétrés de leur devoir, s'occupent de le détruire sans retour, que le royalisme doit espérer de compter dans la République un grand nombre de partisans ». Il ajoute ensuite : «Républicains, Anglomanes de 89, Constitutionnels de 91, le même sort vous est réservé, le même drapeau doit vous réunir ; marchez tous, marchez ensemble pour exterminer les bourreaux qui n'ont d'autre désir que celui de la vengeance » (Moniteur, t. 25, $\mathrm{n}^{\circ}$ 286, 16 messidor an III, p. 126).

(41) Après le décret du 5 prairial an III (24 mai 1795), le Comité de législation fait deux rapports à la Convention sur les députés dénoncés, principalement d'anciens représentants en mission. Le premier rapport a lieu le 13 prairial ( $1^{\mathrm{er}}$ juin 1795) et aboutit à l'arrestation de neuf députés. Le second rapport, le plus important et le moins passionné, a lieu les 21 et 22 thermidor ( 8 et 9 août), dix députés sont arrêtés.

(42) AN D III 346, dossier « Dumont (André) » ; lettre de Pochel, Hautoir (?) près d'Amiens, 4 thermidor (22 juillet). 
du printemps de l'an III. Le contexte politique national joue donc un rôle important dans le rythme de dénonciation mais aussi, dans une moindre mesure, dans le choix des cibles (il n'est en effet pas question de s'attaquer à un réacteur en prairial an III).

L'analyse des dénonciateurs montre que ce sont souvent des personnes qui connaissent bien et le contexte national et le contexte local dans lequel ils évoluent. Cette dernière donnée est importante pour comprendre comment se font les dénonciations car la plupart des conventionnels sont attaqués non à cause de leur position au sein de l'Assemblée mais pour leur action dans telle ou telle localité, soit lors d'une mission soit par l'intermédiaire de fidèles dans le cas des élus du département. La dénonciation a donc un « ancrage géographique » qu'il faut cerner.

\section{Les lieux de la dénonciation : le poids des réalités locales}

Tous les départements n'ont pas connu la même verve dénonciatrice, loin s'en faut. Dans huit départements, aucune lettre n'a été écrite alors qu'environ $30 \%$ des lettres proviennent de seulement sept départements ${ }^{43} \ldots$

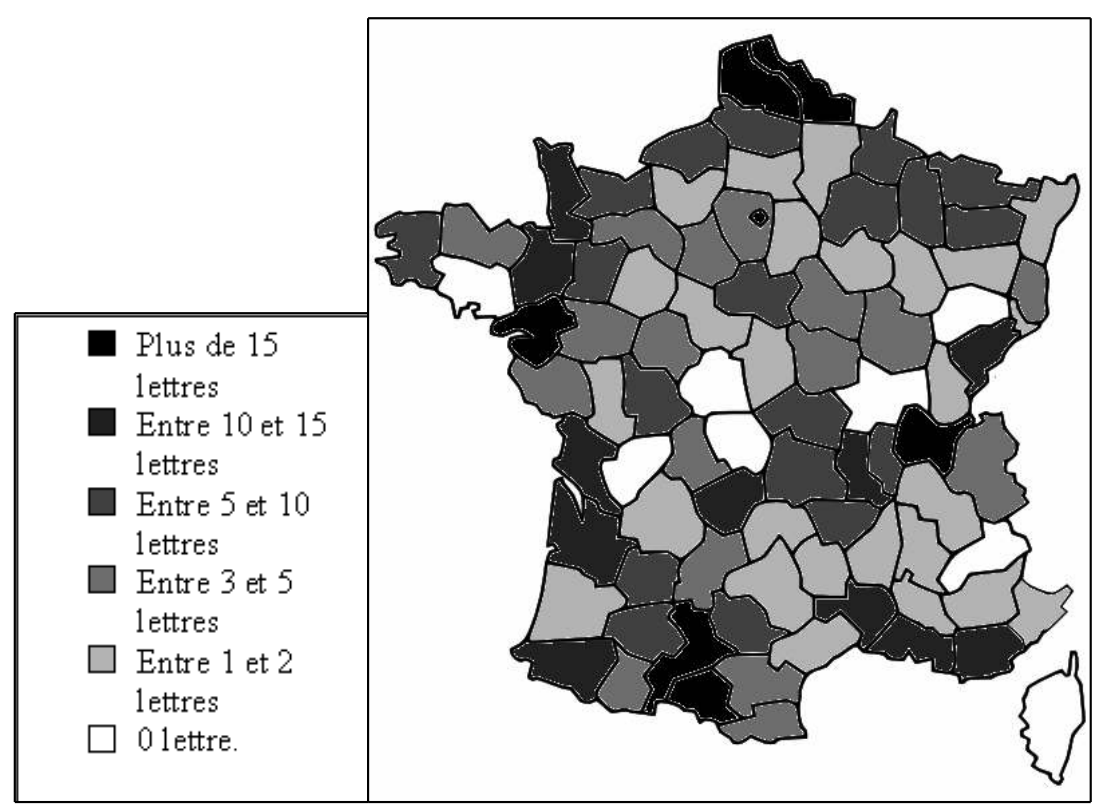

Figure 3. Nombre de dénonciations par département en fonction de l'origine géographique des dénonciateurs.

(43) Sous réserve, bien entendu, de la bonne conservation des archives. 
Cette carte illustre bien la disparité de la pratique dénonciatrice, une disparité dont les causes sont liées à l'histoire de la commune, du district ou du département concernés. La plupart des dénonciateurs en effet, même s'ils réagissent souvent à des événements nationaux, s'inscrivent dans le local ; les faits qu'ils décrivent dans leurs lettres renvoient à ce qu'ils ont vécu ou ce qu'ils ont entendu dire. Rares sont les dénonciateurs qui s'aventurent à mentionner des faits survenus dans des endroits qui ne leur sont pas familiers, sauf en ce qui concerne les prises de position ou l'action au sein de l'Assemblée.

Il est en effet difficile d'analyser la pratique dénonciatrice dans cette France révolutionnaire sans prendre en compte les situations locales. Bien entendu les événements nationaux ont eu des répercussions dans les régions mais la Révolution n'est pas un « bloc »; chaque lieu vit ces événements à sa manière, à son propre rythme. Pourtant les tentatives ne manquent pas pour créer du lien entre les révolutionnaires sur tout le territoire. Les sociétés populaires qui correspondent entre elles, les journaux, les représentants en mission porteurs des ordres de la Convention, les colporteurs, les militants qui parcourent le pays font circuler ces idées, ces rumeurs, qui embrasent Paris, font connaître les journées mémorables (avec souvent une analyse « clef en main »)... L'idéologie révolutionnaire ne s'impose pas, elle se glisse dans la société, s'insinuant souvent dans les conflits préexistants qu'elle contribue à politiser ${ }^{44}$. L'étude de la pratique dénonciatrice donne à voir cette imbrication entre politique nationale et données locales.

\section{L'influence de la violence.}

Une première analyse de la carte montre un lien entre violence révolutionnaire et pratique de la dénonciation. La comparaison de cette carte avec une carte des soulèvements fédéralistes de l'été 1793 ou avec une carte des exécutions en l'an II est éloquente : les régions où la pratique dénonciatrice est la plus forte sont en général les départements qui ont payé le plus lourd tribut à la Terreur. On n'est donc pas surpris de constater que le Gard, les Bouches-du-Rhône ou encore le Var ont été de grands pourvoyeurs de dénonciations puisque ces trois départements ont été le

(44) Jean-Clément Martin donne une synthèse intéressante de la géographie contrerévolutionnaire française, en montrant les limites de toute approche cartographique du phénomène. Toutes les zones de rébellion n'ont pas eu les mêmes moteurs de déclenchement et certaines zones, où l'hostilité à la Révolution était patente, n'ont pu exprimer leur mécontentement du fait d'une répression violente. Voir Jean-Clément MARTIN, Révolution et Contre-Révolution, les rouages de l'histoire, Rennes, Presses universitaires de Rennes, 1996. 
théâtre de violents conflits durant la crise fédéraliste puis d'une terrible répression lors de leur reprise en main par la Convention.

Cependant, si les régions « fédéralistes » sont en général des zones de forte pratique dénonciatrice, ce n'est pas toujours le cas. Ainsi, le département du Calvados, qui a été un centre actif de la résistance après les journées parisiennes de mai et juin 1793, n'est pas un grand pourvoyeur de dénonciations. Il faut dire que la répression qui a suivi la pacification n'a pas eu l'ampleur de celle qui a été conduite à Lyon, Marseille, Toulon ou même Bordeaux, loin de là ${ }^{45}$. Cette modération explique sans doute que les règlements de compte y aient été minimes en l'an III. En revanche la ville de Lyon ne connaît pas une forte pratique dénonciatrice alors que la reprise de la ville par les troupes de la Convention s'est accompagnée de nombreuses arrestations et exécutions ${ }^{46}$. Comment expliquer ce « mutisme » en l'an III ? Première explication : Fouché, qui a conduit la répression avec Collot-d'Herbois, a dû profiter de son passage au ministère de la Police pour faire disparaitre un certain nombre de documents compromettants. De plus, la vigueur de la réaction thermidorienne dans ce département, ainsi que dans toute la vallée du Rhône, a dû détourner les gens de la réaction légale, les uns se terrant de peur d'être attaqués, les autres préférant pourchasser ceux qui les ont persécutés durant la Terreur ${ }^{47} \ldots$ Ce constat vaut aussi sûrement pour les départements bretons touchés par la chouannerie. Une femme prenant la plume en l'an III décrit bien cette atmosphère de peur qui anime une partie des patriotes. Elle déclare vouloir rester dans l'anonymat de peur d'être assassinée par les contre-révolutionnaires ${ }^{48}$... Seuls les bastions républicains bretons éloignés des zones de chouannerie, Saint-Brieuc et Brest principalement, connaissent une certaine pratique dénonciatrice.

(45) Voir Christine PEYRARD, «Le Fédéralisme normand en questions », dans Les Fédéralismes, réalités et représentations, 1789-1874, Actes du colloque de Marseille, septembre 1993, Aix-en-Provence, Publications de l'Université de Provence, 1995, p. 263-270 et Paul R. HANSON, The Jacobin Republic under Fire, the Federalist Revolt in the French Revolution, University Park, PA, The Pennsylvania State University Press, 2003, p. 227-230.

(46) Sur la reconquête de la ville par les troupes de la Convention et la répression qui s'ensuit voir : Michel BIARD, « Collot d'Herbois et la répression à Commune-Affranchie : mythe et réalité », dans Les Fédéralismes, op. cit., p. 207-216 ; id., Collot d'Herbois, légendes noires et Révolution, Lyon, Presses universitaires de Lyon, 1995, p. 125-158.

(47) Sur la Terreur blanche à Lyon et dans la vallée du Rhône, Stephen ClAY, « Vengeance, justice and the reactions in the Revolutionary Midi », French History, février 2009, 23 (1) p. 22-46 ; $i d$., «Les réactions du midi : conflits, continuités et violences », $A H R F, \mathrm{n}^{\circ} 345,2006$, p. 55-91 ; id., «Justice, vengeance et passé révolutionnaire : les crimes de la Terreur Blanche", $A H R F, \mathrm{n}^{\circ} 350,2007$, p. 109-133.. Voir aussi les articles de Bruno BENOÎT, Jean-Louis IsSARTEL, René MouLINAS et Martine LAPIED dans Le Tournant de l'an III, op. cit.

(48) AN D III 356-357, dossier « Ruamps »; sans lieu [Côtes-du-Nord], $1^{\text {er }}$ prairial an II [III] (20 mai 1795). 
L'intensité de la Terreur, ou de la répression des soulèvements contrerévolutionnaires ou fédéralistes, ne suffit souvent pas à expliquer l'ampleur de la pratique dénonciatrice, d'autres facteurs sont à prendre en compte.

L'analyse de la carte montre aussi que la plupart des départements périphériques ont connu une importante pratique dénonciatrice, d'autant plus lorsqu'ils sont proches de frontières où combattent les troupes de la République. Ainsi, alors que les départements du Nord et du Pas-de-Calais sont restés fidèles à la majorité montagnarde durant l'été 1793, la pratique dénonciatrice y est particulièrement forte. De nombreux missionnaires y ont été envoyés ; ils ont contribué à mettre en place une répression sévère justifiée par la position stratégique de la région. Cette sévérité a créé de nombreuses rancœurs au sein d'une population pourtant attachée à la Révolution. La principale cible de ces lettres est le représentant Le Bon, dénoncé dès floréal an II, qui passe pour avoir été l'ami personnel de Robespierre, ce qui en fait une cible de choix en l'an III $^{49}$. D'autres représentants sont néanmoins dénoncés, certains pour leurs accointances avec le royalisme, ce qui montre la division du camp révolutionnaire.

En définitive, si la violence joue un rôle dans l'intensité de la pratique dénonciatrice, elle n'est qu'un des facteurs d'explication parmi d'autres.

\section{Le rôle des divisions locales.}

En Haute-Garonne, un département qui n'a pas pris une part active dans le mouvement fédéraliste mais où la répression a été intense (on est en effet proche de la frontière espagnole), les dénonciations reflètent les divisions de l'opinion locale. Si des terroristes sont dénoncés (Monestier du Puy-de-Dôme, Pinet aîné, Mallarmé...), six lettres s'en prennent également au « réacteur » Laurence qui a effectué une mission dans le Gers, le Tarn et la Haute-Garonne entre germinal et thermidor an III. Durant l'été 1795, Laurence est dénoncé par plusieurs Toulousains tandis que les habitants de Revel font son éloge tout en reprochant à Calès de tout faire pour maintenir en place les jacobins locaux... La Haute-Garonne est en effet un département où le jacobinisme reste solidement implanté jusqu'en mai 1795 mais où la Terreur blanche s'est également manifestée ${ }^{50}$, sans pour autant tomber

(49) Sur la mission de Le Bon dans le Nord et le Pas-de-Calais voir Louis JACOB, Joseph Le Bon, 1765-1795. La Terreur à la frontière (Nord et Pas de Calais), Paris, Mellottée, n. d. (1933 ou 1934), 2 vol.

(50) À Toulouse le journal l'Anti-terroriste, qui paraît en l'an III, est l'un des journaux contre-révolutionnaires les plus virulents de France. Jacques GODECHOT, « La Contre-Révolution 
dans des excès sanglants ${ }^{51}$, ce qui explique cette expression simultanée d'opinions si contraires.

Dans l'Ain, où la résistance aux Montagnards a été forte durant l'été 1793, la lecture des dénonciations montre la division profonde du camp révolutionnaire. À son arrivée en décembre, le représentant Gouly reçoit le soutien des éléments les plus modérés et s'oppose aux « sans-culottes » radicaux, qui ont fui à Paris durant la crise fédéraliste. Ces derniers dénoncent à la Convention la « contre-révolution » que le représentant et ses amis font régner dans le département. L'arrivée d'Albitte, qui remplace son collègue en janvier, bouscule les équilibres puisqu'il s'appuie sur les plus radicaux. Après le 9 Thermidor, le rapport de force change à nouveau ; les « modérés » sortent de prison et prennent la plume ${ }^{52}$.

Le représentant envoyé en mission dans les départements est en effet souvent obligé de s'appuyer sur les militants locaux qui connaissent mieux que lui la politique locale. Dans certaines régions plusieurs groupes s'affrontent et le représentant qui prend parti pour l'un d'eux, s'expose à la haine des autres ${ }^{53}$. Parfois même, ces partis antagonistes tirent parti de la division au sein d'une équipe missionnaire ${ }^{54}$, chaque camp essayant de s'appuyer sur un protecteur. Car la politique se calque souvent sur des conflits plus anciens.

\section{Des facteurs d'explication variés qui se combinent.}

Plusieurs facteurs peuvent donc se combiner pour expliquer la pratique dénonciatrice d'un département. Certains relèvent de l'histoire nationale, d'autres tiennent à des facteurs plus locaux ou même personnels. Les départements de la Loire-Inférieure, de l'Ille-et-Vilaine et de la Manche

dans le Midi toulousain », dans Les Résistances à la Révolution, actes du colloque de Rennes (17-21 septembre 1985), Paris, Éditions Imago, 1987, p. 122.

(51) Georges FOURNIER, « Réalités et limites de la réaction thermidorienne dans l'Hérault, l'Aude et la Haute-Garonne », dans Le Tournant de l'an III , op. cit., p. 491.

(52) Voir Jérôme CROYET, « La Mission du conventionnel Albitte dans l'Ain (28 nivôse an II-11 floréal an II) », dans Germain SICARD (dir.), Justice et Politique : la Terreur dans la Révolution française. Études d'histoire du droit et des idées politiques, Toulouse, Université des Sciences-sociales de Toulouse, $\mathrm{n}^{\circ} 1,1997$, p. 155-167 ; et aussi Louis MEUNIER, « Albitte, conventionnel en mission (Ain et Mont-Blanc) », AHRF, 1946, p. 49-66 et 238-277.

(53) Voir Michel BIARD, Missionnaires de la République, op. cit., p. 265.

(54) En Lorraine par exemple les divisions au sein des révolutionnaires sont aggravées par les dissensions au sein de l'équipe missionnaire. Voir Olivier VINCIENNE, «Les Démêlés de Faure et de Lacoste représentants en mission dans les départements lorrains, 1793-1794 », Pays lorrain, 61 ${ }^{\mathrm{e}}$ année, 1980, p. 43-48. 
présentent de nombreuses similitudes ${ }^{55}$. La forte pratique dénonciatrice qui marque ces départements est certainement due à la personnalité des représentants qui y ont été envoyés : Carrier à Nantes ${ }^{56}$ ou Le Carpentier sur la côte de la Manche ${ }^{57}$.

Pourtant, comme le montre le cas de la Corrèze, même un département en apparence calme peut connaître une forte pratique dénonciatrice. Restée fidèle à la majorité montagnarde après juin 1793, la Corrèze a été relativement épargnée par les rigueurs terroristes. Quatorze dénonciations y sont pourtant rédigées et elles proviennent toutes de Brive et du district d'Ussel. Dans ce dernier cas c'est une émeute locale en frimaire an $\mathrm{II}^{58}$, ayant conduit à la mise à mort de quatre personnes, qui a cristallisé les oppositions. Lanot, qui était alors en mission, est accusé d'avoir mené une répression inique et d'avoir fait condamner des patriotes. L'ampleur du mouvement dénonciateur s'explique par l'attitude du procureur syndic du district d'Ussel qui invite, par une lettre circulaire du 28 floréal an III (17 mai 1795), tous les citoyens à formuler leurs plaintes à propos de cette émeute de Maymac ${ }^{59}$. On voit ici qu'un événement local joint à la volonté d'un administrateur zélé explique le mouvement de dénonciations dans le département.

Bien souvent, le nombre de dénonciations écrites dans un département dépend tout autant du vécu que de la capacité à mobiliser la population dans l'acte de dénonciation.

La répétition dans un grand nombre de ces lettres de formules éculées, mille fois entendues, ne doit pas faire oublier que la dénonciation, loin d'être seulement un acte d'allégeance au pouvoir en place (ou un acte de contestation), est aussi un récit vivant qui permet souvent d'appréhender la grande variété de réactions qu'a suscitée la Révolution dans les départements. L'analyse de cette carte de la pratique dénonciatrice

(55) Tous les trois ont participé au mouvement fédéraliste et sont marqués par de forts mouvements contre-révolutionnaires ; de plus, ils sont tous situés sur des zones frontières stratégiques (la Convention craint en permanence un débarquement anglais dans ces zones peu sûres).

(56) Sur les vingt lettres s'attaquant à Carrier, quatorze ont été écrites par des personnes originaires de Nantes.

(57) Sur les vingt-deux lettres qui ont été envoyées pour le dénoncer, neuf proviennent de l'Ille-et-Vilaine et onze de la Manche, département dont il est originaire. Sur sa mission, voir Jean-Louis MENARD, Jean-Baptiste Le Carpentier, délégué par la Convention nationale dans les départements de la Manche et autres environnants, Bricquebosc, Éditions des Champs, 2001.

(58) Voir François ARSAC, «Une Émeute contre-révolutionnaire à Meymac (Corrèze) », $A H R F, \mathrm{n}^{\circ} 13$ (1936), p. 149-162.

(59) Ainsi, sur les quatorze lettres qui ont été envoyées à la Convention pour dénoncer Lanot, onze ont été expressément rédigées suite à la lettre circulaire du procureur-syndic du district d'Ussel et une autre semble avoir été inspirée par cette même circulaire. AN D III 352, « dossier Lanot ». 
montre ainsi que ce sont les situations locales qui expliquent le mieux les différences d'intensité entre deux régions. Ainsi un département où plusieurs partis s'affrontent, avec ou non le soutien de représentants, a plus de chances de voir émerger un grand nombre de dénonciations à l'instar de la Haute-Garonne, ou de l'Ain. À l'inverse, les régions où domine un seul groupe, qu'il soit partisan ou adversaire acharné de la Révolution, ont moins de chance de voir surgir des dénonciations : soit parce que la Révolution n'y a pas laissé de plaies profondes (par exemple la Creuse ou l'Aveyron), soit encore parce que la peur paralyse les populations (c'est le cas notamment dans les zones de chouannerie et en Vendée).

La dénonciation donne donc souvent à voir cette articulation entre le local et le national. Les dénonciateurs sont des personnes - des hommes ${ }^{60}$ - qui sont à la fois très impliqués dans leur localité et très au fait des événements nationaux. Beaucoup de dénonciateurs sont en effet membres d'administrations, militaires, de sociétés populaires ${ }^{61} \ldots$ La dénonciation concerne avant tout les élites politiques de cette France révolutionnaire ${ }^{62}$ et ne concerne que marginalement le petit peuple, sauf sa frange la plus politisée bien entendu.

Après la chute des «triumvirs », les conventionnels doivent trouver des boucs émissaires pour satisfaire une opinion désireuse de justice, de vengeance, au moment où les suspects sortent des geôles. Carrier est le premier conventionnel « sacrifié ».

La plupart des conventionnels savent en effet qu'ils sont tous, à des degrés divers, impliqués dans la Terreur mais les exigences politiques de l'an III, et des considérations personnelles, ne permettent pas à l'Assemblée de faire un mea culpa général qui, pense-t-elle, ferait le jeu de la ContreRévolution. Car si la France révolutionnaire paraît victorieuse sur tous les fronts après la chute de Robespierre, le pays n'en reste pas moins fragile comme en témoigne la reprise des troubles durant le printemps de l'an III. La pacification, pour ces hommes politiques, passe donc par une réaction qu'ils s'efforcent, tant bien que mal, de contrôler. Les députés, cherchant comme leurs prédécesseurs à « finir la Révolution », ne peuvent

(60) On ne trouve en effet que six femmes parmi les cent soixante-treize dénonciateurs individuels.

(61) $41 \%$ du total des lettres sont l'œuvre de personnes agissant seules. $15 \%$ du total a été rédigé par des administrations (administrations départementales, de district, municipales ou encore comités de surveillance) et $13 \%$ par des sociétés populaires.

(62) La dénonciation est une pratique purement révolutionnaire. Les contre-révolutionnaires notoires (qui s'affichent comme tels) ne prennent que très rarement la plume. 
faire l'économie d'une remise en cause de leur propre histoire. Ils forgent alors une explication simpliste des événements qu'ils viennent de vivre : Robespierre et ses complices (aussi bien dans l'Assemblée que dans les autres administrations, parisiennes ou régionales) sont les uniques responsables de la politique terroriste, leur punition et le rappel au pouvoir de leurs anciennes victimes (notamment les girondins mais aussi les émeutiers vendéens ou chouans «égarés ») doivent permettre de remettre en ordre le pays. Au printemps, les émeutes donnent un nouvel élan à cette épuration qui ne prend fin qu'avec le vote de la Constitution.

Cette chronologie nationale a grandement conditionné la pratique dénonciatrice en l'an III, les dénonciateurs suivent de près les événements nationaux et réagissent aux changements qui affectent la Convention. Cependant, ces hommes qui prennent la plume sont la plupart du temps des acteurs locaux de la Révolution, soit parce qu'ils ont joué un rôle durant la Terreur, soit parce que la Réaction leur permet de retrouver la place qu'ils avaient perdue. Ces lettres témoignent de cette imbrication entre le politique et les réalités locales, elles montrent la façon dont les acteurs révolutionnaires se réapproprient la chronologie révolutionnaire à l'aune de leur expérience locale.

Les dénonciations sont un des aspects de cette Réaction thermidorienne, elles donnent non seulement à voir les pratiques terroristes et leur perception mais aussi comment, dans les départements, on a essayé de « sortir de la Terreur ». Selon les régions, la Réaction thermidorienne a pu prendre des formes variées, souvent plus douces que la « Terreur blanche » qui sévit dans le Midi. En ce sens il vaudrait mieux parler de réactions thermidoriennes plutôt que de Réaction thermidorienne.

Jean-Baptiste LEGOFF Archiviste-Paléographe Archives départementales de la Meuse 26 rue d'Aulnois - B.P. 514 - 55012 Bar-le-Duc Cedex jblgff@gmail.com 